INTERNATIONAL JOURNAL OF MULTIDISCIPLINARY RESEARCH AND ANALySis

ISSN(print): 2643-9840, ISSN(online): 2643-9875

Volume 05 Issue 02 February 2022

DOI: 10.47191/ijmra/v5-i2-33, Impact Factor: 6.072

Page No. 466-471

\title{
Mental Health during COVID-19 Pandemic and its Effects on Migrant Laborers, Women and Adolescents in Southeast Asia:
}

\section{A Qualitative Review}

\author{
Narayana Prasad MD, MPH, MS, ALM ${ }^{1}$ Sloka lyengar Ph.D. PMP², Hanifa M. Denny MPH, \\ Ph.D. ${ }^{3}$, Yasmine Aulia Gunawan M.Sc. ${ }^{4}$, Raviteja Innamuri DPM, MD ${ }^{5}$, Sharad Phillip MD ${ }^{6}$, \\ Oluranti Samuel Dphil ${ }^{7}$ \\ ${ }^{1}$ Technical Director and co-founder of Public Health Literacy, Boston USA \\ ${ }^{2}$ Scientist, American Museum of Natural History, New York, USA \\ ${ }^{3}$ Associate Professor of Public Health and Dean of Academic, students and Alumni Affairs, Diponegoro University, Indonesia \\ ${ }^{4}$ Doctoral Student Department of Healthcare Administration, Major in Psychology, Asia University, Taiwan \\ ${ }^{5}$ Assistant Professor of Psychiatry, Government Medical College and General Hospital, Nizamabad, Telangana, India \\ ${ }^{6}$ Consultant Psychiatrist, Mindful Rejuvenation Hospital and Mindful Kids Care Services, Kochi, Kerala, India \\ ${ }^{7}$ Senior Lecturer, Faculty of Social sciences, Lagos state University, Nigeria
}

\begin{abstract}
Objective: The COVID-19 pandemic has affected the physical, mental, and social well-being of millions of people across the globe. This paper will discuss the impact of COVID-19 on preexisting and emerging mental health issues amongst various subgroups of the vulnerable population in Southeast Asia.

Methods: Qualitative review of existing knowledge on mental health issues during COVID-19 pandemic among migrant laborers, women and adolescents.

Conclusion: Given the profound impact of COVID -19 beyond physical health, policy makers, scientific communities and faithbased institutions should make every effort to turn the COVID-19 crisis into an opportunity by developing a strong and robust consensus that is based on building public trust in partnership to provide societal mental health, which is an unappreciated asset of a nation. We conclude that while there is considerable amount of work in the field of mental health has been done, many efforts are siloed, and do not consider the unique cultural characteristics involved. In addition to providing ventilators and acute care to COVID-19 affected patients at the hospitals, we need to design policies to allocate resources for the post-pandemic burden on our communities and develop multi-disciplinary approaches. We suggest that these approaches should become routine in healthcare settings and should be appropriate to the individual's cultural context. We argue that the generation of robust data, scientific evidence, and multi-disciplinary resources-sharing are required that would necessitate to produce the interventions that are culturally specific and scalable.
\end{abstract}

KEYWORDS: COVID 19, mental health, vulnerable population

\section{BACKGROUND}

Dr. Benjamin Miller, chief strategic officer at Well Being Trust foundation, USA who works with Graham Center for Policy Studies in Family Medicine and Primary Care says, "COVID-19 has come in as a multiplier of social, economic factors that is making deaths of despair even worse". So called word, "deaths of despair", coined for the deaths by suicide, or associated with alcohol and drug use that are related to socio-economic fallout. George Everly PhD, a psychologist from Johns Hopkins School of Medicine suggests the long-term consequences of the pandemic and ensuing mental issues typically begin weeks or months after the pandemic's peak of infections, when deaths of despair usually rise. Unfortunately, the recurring waves of the pandemic are leaving with little time to address or note these deaths of despair. Nearly 182,000 people died from deaths of despair in $2019 .{ }^{1}$ Some predictive models indicate additional 75,000 additional deaths during to COVID-19 pandemic and continued paucity of mental services. The 


\section{Mental Health during COVID-19 Pandemic and its Effects on Migrant Laborers, Women and Adolescents in Southeast Asia: A Qualitative Review}

stakes could not be higher. If not adequately or appropriately addressed, the mental health consequences for a generation of children and young people could far surpass the immediate health and economic impact of the COVID-19 pandemic, leaving longterm social and economic consequences in its wake. ${ }^{2}$

This paper aims to explore the prevalence of mental health issues in vulnerable populations that are either exaggerated or unmasked by COVID-19 and gives a bird's eye view on how social determinants play in a specific Southeast Asian context. Although many of the mental health issues are applicable to various degrees of severity to various vulnerable population, this paper will focus on migrant laborers, women and children and adolescents.

\section{MENTAL HEALTH IN GENERAL}

"There is no health without mental health." - the World Health Organization (WHO) proclaimed this sentiment in 2018, which has become profoundly striking in the times of the pandemic. ${ }^{3}$ Lester Breslow, a highly acclaimed public health expert who has done notable work on measuring health, declared the future of health - it is not just preventing and treating diseases; it is about expanding social resources for mental health. ${ }^{4} \mathrm{He}$ underscored that health equity challenges would require caregivers to rethink their assumptions, and see the health through the lens of communities. ${ }^{5}$ According to WHO, mental well-being is more than the absence of mental illness. Without a long term coordinated mental health policy, there will be debilitating effects on the majority of vulnerable population. ${ }^{6}$

"There are known knowns. there are known unknowns. But there are also unknown unknowns, the ones we don't know we don't know, ${ }^{7}$ according to Former Secretary of Defense Donald Rumsfeld. Likewise, the unknowns in terms of public health, and its implications will keep piling up to reveal themselves over time. COVID-19 has created a greater deal of stigma, mainly due to a lack of awareness about how the disease spreads and attacks the human body. As with any community trauma, the most vulnerable populations of a community have suffered the most negative impacts during the COVID-19 pandemic. Investing in improving mental health increases productivity by gaining more robust personal relationships and a sense of belonging. ${ }^{5}$

Mental health is difficult to describe and define - the definitions of poor mental health, mental illness, and mental wellbeing are not consistent among populations, and even between people within a population. ${ }^{8} \mathrm{It}$ is possible that someone with poor mental health may never be clinically diagnosed with mental illness. There are several definitions of mental health; specifically, the WHO definition of mental health revolves "a state of well-being in which the individual realizes his or her own abilities, can cope with normal stresses of life", 9,10 but misses an additional element of effects of dynamic nature of mental health that revolves around the human experiences to cope with the adverse life events and maintain state of internal equilibrium. ${ }^{11} \mathrm{To}$ assess dynamic nature of mental health, the existing diagnostic methodology will need to be redefined. In contrast to physical conditions, where determining cause-and-effect can be straightforward, mental health conditions present a challenge at that front. This is partly because of legal, economic, insurance justifications that are made in compliance with the Diagnostic and Statistical Manual of Mental Disorders (DSM). ${ }^{12}$ One definition of mental health revolves around the concepts of self-realization, mastery over the environment, and autonomy but this view has had criticisms for being overly Western-centric. ${ }^{10}$ As can be expected, sociocultural factors come into play, as do differences in perception that is not uncommon when it comes to seeking help for mental health issues. The Mental Illness Stigma Framework (MISF) has provided a structural foundation to define stigma surrounding mental illnesses, and has concluded that while some aspects of stigma such as stereotyping and discrimination have been somewhat studied, others that describe the internal state of the person with mental illness (e.g., internalized stigma) have been studied less so. ${ }^{13}$ Perspectives of the stigmatizer and the stigmatized need to be considered, as they may vary depending on the culture and other surrounding factors. ${ }^{14}$ Given the rootedness of mental health in a specific cultural setting, social cohesion play a stronger role in mental health perception. This will define available resources and help-seeking strategies, the as well as community support. ${ }^{15}$ These aspects come into play when we think of a heavily community-oriented cultures (e.g., some countries in Southeast Asia) vs. cultures that are perceived more individualistic (e.g. some cultures in the Global North). This leads us to wonder if there can ever be a universal understanding of mental health and mental well-being. Ethnic practices, regional culture play a role when thinking about mental health for vulnerable people within a given community. ${ }^{16}$ Given that diverse ethnic minorities in the various countries across the globe, makes acceptability of the definition and management of mental health illnesses one think of the challenging, although basic physiological components of mental health recovery that are universal ${ }^{17}$ This brings us to another facet of mental health, which is that the state of a person's mental wellbeing can change over time, which means that help-seeking strategies, and support required also change over time. The intersection of mental health with age, ability, sexual orientation, gender identity, and race are well-documented. ${ }^{18}$ The intersectional role of factors in mental health also means that there are underlying intersectional inequalities beyond mental health. ${ }^{19}$ 


\section{Mental Health during COVID-19 Pandemic and its Effects on Migrant Laborers, Women and Adolescents in Southeast}

Asia: A Qualitative Review

Mental well-being is essential to maintain personal well-being. There is an intricate link between mental and physical health, so that mental illnesses can negatively impact a person's ability to maintain positive health. Chronic physical disorders such as diabetes and hypertension are often associated with poor mental health, furthering the connection between mental and physical health. ${ }^{20}$ Resilience, colloquially termed as "bouncing back" can be defined as the capability to "adapt well in the face of adversity, trauma, threats, and significant sources of stress". ${ }^{21} .{ }^{22,}{ }^{23}$ While it is not easy to predict who will go on to develop a mental illness, there are specific populations that are at a greater risk of mental illness. ${ }^{24}$ There are protective factors when it comes to mental health and wellbeing, and while we cannot prevent mental illnesses, a culture of promoting mental well-being starts in childhood/adolescence; this is because of the impact of early childhood experiences can lead to mental disorders later in life. While there are nuances, individuals with a history of multiple childhood adversities are more susceptible to common mental disorders later in life. ${ }^{21,22,23}$ Studies have found that childhood maltreatment in a specific population increases risk of major depressive disorder (MDD), generalized anxiety disorder (GAD) and drug use disorder (DUD)..$^{25}$ In short, mental health is an enormously complicated topic with varied facets. The determinants of mental health and wellbeing are many, e.g., age., gender identity, caste, economic status, and stigma. ${ }^{26}$ The real brunt of a catastrophic event such as COVID-19 is always felt by vulnerable populations due to socio-economic conditions preceding and succeeding the pandemic. The dehumanizing effects of a massively traumatic event like COVID-19, as well as PTSD, depressive and anxiety disorders, have exposed hidden mental issues that otherwise would have been ignored. ${ }^{27,28}$

\section{MIGRANT LABORERS}

The migrant workforce is one of the vulnerable population sectors that are receiving the brunt of COVID-19 and more susceptible to exaggerated mental health conditions. The definition of migrant labor has been vague and represents a complex combination of caste/race, communal segregation that has been variously called based on the region of origin such as "Yanadi" in Andhra Pradesh, State of Southeast India, "Dalit" from Haryana, Northeast part of India. ${ }^{29}$ The sudden decision by the South-east Asian countries to impose a nationwide lockdown within hours came with massive, unintended, and catastrophic consequences to the migrant labor that make up informal economy in South east Asian countries. . The sudden lockdown policy stranded and led to the massive overnight employment of these daily wage laborers. The conservative estimate of unemployment rate was $23 \%$ in India. ${ }^{30}$ The International Labor Organization (ILO) estimated that 400 million informal laborers would be pushed to worsening poverty due to COVID-19. ${ }^{31}$ In Indonesia, there was a sudden surge in anxiety disorder among single and non-married migrant workers; the nuance here is that Indonesian migrant workers who are single or have no family support are likely to suffer from anxiety. This sudden increase in anxiety was more prevalent among people with low educational attainment. Similar findings were observed among farmers, fishermen and other low income labor population who are predominantly migrants. ${ }^{32}$ Although there is no computed data on mental health among migrant labor forces, uncertainty about their job prospects, fear of deportation, fear of lack of resources to support themselves and loved ones had put them in a more vulnerable position to suffer from mental health issues. According to Nelson Vinod Moses, Founder of Suicide Prevention India Foundation, there has been substantial evidence of increased prevalence of suicides and other mental health disorders in rural India due to massive return of migrant workers and the farming community. ${ }^{33}$

\section{WOMEN}

Southeast Asian countries rank one of the lowest women workforce participation rates in the world. In addition, the lockdown has affected predominantly women primarily because of social isolation and domestic violence. ${ }^{34}$ In the broader discourse on public health, women, both maternal physical and mental health, are usually overlooked. It is estimated that nearly 35 percent of women worldwide suffer from depression during pregnancy and postpartum. Pregnant women and new mothers are at an elevated risk of suffering from mental health problems. It has been observed that the uncertainty surrounding COVID-19 has led to higher levels of depression among women during and after pregnancy. Pregnant women may feel social isolation and have a greater fear of infection for themselves, as well as their infants. Lack of health facilities and increasing number of home deliveries, without the assistance of trained health workers could have heightened the distress and depression in these women. Gender-based exclusion and denial of the right to express themselves have intensified during COVID-19. Numerous studies have shown the effects of COVID-19 in exaggerating prevalence of depression and anxiety in the perinatal period. ${ }^{35}$ In addition, here is evidence in increased cases of domestic violence on women. ${ }^{36}$ The domestic violence encompasses physical and psychological violence although the later often remains undetected. Countries like Bangladesh and other southeast Asian countries saw increase in intimate partner violence also called "wife beating" during lockdown period of pandemic. The implicit nature of the challenge is there is lack of safe place to call the helplines despite availability of numerous call centers and helplines, access to smartphones. ${ }^{37,38}$ 


\section{Mental Health during COVID-19 Pandemic and its Effects on Migrant Laborers, Women and Adolescents in Southeast}

Asia: A Qualitative Review

Speaking to differences even among individuals in the broader group of women, elderly women and widows continue to be more affected by the COVID-19 pandemic. Given that women take up most of the caregiving burden in households, women worldwide have suffered from those responsibilities in the face of a lack of access to childcare due to social distancing and isolation. In India, patriarchy, child marriage, quest for a male child, living with in-laws, "joint family", widowhood, lack of rights, domestic violence, effect of role transition, dowry deaths, etc. Depression as the most common women's mental health problem. ${ }^{39}$ In Taiwan one estimate suggests that $78 \%$ of Taiwanese women have had an episode of depression, and that this risk was greater in immigrant Taiwanese women. Domestic violence and ensuing post-traumatic stress syndrome, family pressures, negative interactions between family members have all been suggested for this phenomenon in Taiwanese women. ${ }^{40} 4142$

\section{ADOLESCENTS}

Mental health disorders are the leading factor of disease burden among people between ages of 10-24 years. Additionally, those living in poverty are twice likely to suffer from mental health problems compared to average income population. ${ }^{43}$ Children and adolescents suffer from isolation and loneliness with increased the risk of depression, as well as the possibility of anxiety at the time of loneliness. Marginalized children and adolescents are believed to be susceptible to mental health risk. ${ }^{44}$ Several mental health problems in Indonesian adolescents such as anxiety and sleep deprivation were also found in the early of pandemic. ${ }^{34}$ The duration of loneliness was strongly correlated with mental health symptoms than the intensity of loneliness. The investigators found young people were as much as three times more likely to develop depression in the future due to social isolation, with the impact of loneliness on mental health lasting up to nine years later. ${ }^{45}$ In addition, young people living in poverty suffer from multiple disadvantages including violence, lack of basic educational and employment needs and hence limited future life opportunities and put them in poorer mental health. ${ }^{46}$ Monetary poverty is associated with increased risk of depressive disorders in young people. ${ }^{47}$ Additionally, such mental health deterioration among young people has pushed them to worsened poverty due to increased healthcare spending and loss of already-compromised employment. Social causation and social drift reinforces poverty and depression cycles. ${ }^{48}$ During the quarantine period in the Philippines, researchers reported that adolescence females tended to have poorer mental health status compared to other populations. Deprivation of necessities for wellbeing that is exaggerated by pandemic is highly associated with depressive disorders among young primarily triggered by uncertainty. This calls for further understanding of underlying factors that are either contributing or not contributing the mental health crisis. ${ }^{47}$

\section{LIMITATION OF THE PAPER}

This paper does not address clinically diagnosed mental illnesses and identity-based exclusion during COVID 19. The mental illnesses related to inadequate basic physical amenities such as water, latrine facilities are not addressed in this paper. The Southeast Asian geographies were chosen because of the authors' affiliations and experiences. In order to retain the focus of the paper, we have limited our commentary to few vulnerable populations. Migrant laborers, women and adolescents were discussed as they constitute a vast majority of the population. However, we do not suggest that each of these groups is a monolith, so even within each group, there are specific considerations such as single women, adolescent women, married women, and those that have been widowed. While we have not discussed at each of these segments in specific, there are intersectionality and overarching principles that can guide our understanding of the mental well-being. Additional work is needed to study and address the mental health consequences of these diverse and varied populations and topics.

\section{CONCLUSION}

The COVID 19 crisis in southeast Asia has brought a century old discussion of mental health of individuals and communities to the forefront. Given the situation where the world stands amidst, global pandemic, economic slowdown, and unaddressed mental health crisis, a multi-disciplinary and multi-stakeholders collaboration to address mental health is essential. Given the profound impact of COVID -19 beyond physical health, policy makers, scientific communities and faith-based institutions should make every effort to turn the COVID-19 crisis into an opportunity by developing a strong and robust consensus that is based on building public trust in partnership to provide societal mental health, unappreciated asset of a nation. Most importantly, all initiatives for improving mental health should integrate empowerment of the most vulnerable groups and social integration. In addition to providing ventilators and acute care to COVID-19 affected patients at the hospitals, we need to design policies to allocate resources for the post-pandemic burden on our communities and develop multi-disciplinary approaches. We suggest that these approaches should become routine in healthcare settings and should be appropriate to the individual's cultural context. We argue that the generation of robust data, scientific evidence, and multi-disciplinary resources-sharing are required that would necessitate to produce the interventions that are culturally specific and scalable. 
Mental Health during COVID-19 Pandemic and its Effects on Migrant Laborers, Women and Adolescents in Southeast Asia: A Qualitative Review

\section{REFERENCES}

1) After record 'deaths of despair' in 2019, early pandemic data is grim. Available at: https://www.newsnationnow.com/usnews/after-record-deaths-of-despair-in-2019-early-pandemic-data-is-grim/. Accessed February 13.

2) Coronavirus triggers mental health crisis in India. Available at: https://www.dw.com/en/coronavirus-triggers-mentalhealth-crisis-in-india/a-54011738.

3) Kolappa K, Henderson DC, Kishore SP. No physical health without mental health: lessons unlearned? Bulletin of the World Health Organization. 2013;91:3-3A.

4) Breslow L. An interview with Dr. Lester Breslow [interview by JoAna Stallworth and Jeffery L. Lennon] American journal of public health. 2003;93:1803-1805.

5) Reinventing Healthy Communities: Implications for Individual and Societal Well-Being. Mar JD, editor2016.

6) Economic and Political Weekly. Gurgaon: 2020.

7) 'Nothing ever ends': Sorting through Rumsfeld's knowns and unknowns. Available at: https://www.washingtonpost.com/lifestyle/style/rumsfeld-dead-words-known-unknowns/2021/07/01/831175c2-d9df11eb-bb9e-70fda8c37057_story.html.

8) About Mental Health. Available at: https://www.cdc.gov/mentalhealth/learn/index.htm. Accessed February 13.

9) Mental health: strengthening our response. Available at: https://www.who.int/news-room/fact-sheets/detail/mentalhealth-strengthening-our-response. Accessed February 13.

10) Galderisi S, Heinz A, Kastrup M, Beezhold J, Sartorius N. Toward a new definition of mental health World psychiatry : official journal of the World Psychiatric Association (WPA). 2015;14:231-233.

11) Galderisi S, Heinz A, Kastrup M, Beezhold J, Sartorius N. A proposed new definition of mental health Psychiatr Pol. 2017 Jun 18;51:407-411.

12) Reese H. The Real Problems With Psychiatry. Available at: https://www.theatlantic.com/health/archive/2013/05/thereal-problems-with-psychiatry/275371/. Accessed February 13.

13) Fox AB, Earnshaw VA, Taverna EC, Vogt D. Conceptualizing and Measuring Mental Illness Stigma: The Mental IIIness Stigma Framework and Critical Review of Measures Stigma Health. 2018 Nov;3:348-376.

14) Mental Illness Stigma Framework. Available at: https://earnshawlab.org/mental-illness-stigma-framework/. Accessed February 13.

15) Four Ways Culture Impacts Mental Health. Available at: https://www.mentalhealthfirstaid.org/2019/07/four-waysculture-impacts-mental-health/. Accessed February 13.

16) Canino G, Alegria M. Psychiatric diagnosis - Is it universal or relative to culture? Journal of child psychology and psychiatry, and allied disciplines. 2008 04/01;49:237-250.

17) Moran G, Russo-Netzer P. Understanding Universal Elements in Mental Health Recovery: A Cross-Examination of Peer Providers and a Non-Clinical Sample Qualitative Health Research. 2015 2016/01/01;26:273-287.

18) Miranda Massie WWPaLC, UBC. A faciliator's guide: Intersectional Approaches to Mental Health Education. 2020.

19) Fagrell Trygg N, Gustafsson PE, Månsdotter A. Languishing in the crossroad? A scoping review of intersectional inequalities in mental health International Journal for Equity in Health. 2019 2019/07/24;18:115.

20) Ducat L, Philipson LH, Anderson BJ. The mental health comorbidities of diabetes JAMA. 2014;312:691-692.

21) Building your resilience. Available at: https://www.apa.org/topics/resilience. Accessed February 13.

22) Hyun HS, Kim MJ, Lee JH. Factors Associated With Post-traumatic Growth Among Healthcare Workers Who Experienced the Outbreak of MERS Virus in South Korea: A Mixed-Method Study Frontiers in Psychology. 2021 2021-April-22;12. Original Research

23) Ogińska-Bulik N, Kobylarczyk M. Association between resiliency and posttraumatic growth in firefighters: the role of stress appraisal Int J Occup Saf Ergon. 2016;22:40-48.

24) Mental Health and Mental Disorders. Available at: https://www.healthypeople.gov/2020/topics-objectives/topic /mental-health-and-mental-disorders. Accessed February 13.

25) Myers B, Bantjes J, Lochner C, Mortier P, Kessler RC, Stein DJ. Maltreatment during childhood and risk for common mental disorders among first year university students in South Africa Social Psychiatry and Psychiatric Epidemiology. 2021 2021/07/01;56:1175-1187.

26) Alegría M, NeMoyer A, Falgàs Bagué I, Wang $Y$, Alvarez K. Social Determinants of Mental Health: Where We Are and Where We Need to Go Current psychiatry reports. 2018;20:95-95. 
27) Bracken PJ, Giller JE, Summerfield D. Psychological responses to war and atrocity: The limitations of current concepts Social Science \& Medicine. 1995 1995/04/01/;40:1073-1082.

28) Summerfield D. A critique of seven assumptions behind psychological trauma programmes in war-affected areas Social Science \& Medicine. 1999 1999/05/01/;48:1449-1462.

29) 'Covid-19 Reaffirmed India's Caste, Class Inequalities': Author Suraj Yengde on Learning Inclusion from Ambedkar. Available at: https://www.news18.com/news/india/coronavirus-reaffirmed-indias-caste-class-inequalities-author-surajyengde-on-inclusion-amid-a-pandemic-2577035.html. Accessed February 13.

30) Vyas M. The jobs bloodbath of April 2020. Available at: https://www.cmie.com/kommon/bin/sr.php? kall=warticle $\& d t=2020-05-05 \% 2008: 22: 21 \& m s e c=776$. Accessed February 13.

31) ILO Monitor: COVID-19 and the world of work. Fifth edition International Labor Organization 2020.

32) Megatsari $H$, Laksono AD, Ibad M, Herwanto $Y T$, Sarweni KP, Geno RAP, et al. The community psychosocial burden during the COVID-19 pandemic in Indonesia Heliyon. 2020 Oct;6:e05136.

33) https://www.hindustantimes.com/analysis/covid-19-india-is-staring-at-a-mental-health-crisis/storyhmBOzUYsbo3SmtIW ilmBzL.html.

34) Covid-19: India is staring at a mental health crisis. Available at: https://www.hindustantimes.com/analysis/covid-19india-is-staring-at-a-mental-health-crisis/story-hmBOzUYsbo3SmtlWilmBzL.html. Accessed February 13.

35) Muhammad Iqbal LR. Deteksi Dini Kesehatan Mental Akibat Pandemi Covid-19 Pada Unnes Sex Care Community Melalui Metode Self Reporting Questionnaire.

36) Bachani S, Sahoo SM, Nagendrappa S, Dabral A, Chandra P. Anxiety and depression among women with COVID-19 infection during childbirth-experience from a tertiary care academic center AJOG Glob Rep. 2022 Feb;2:100033.

37) COVID-19, Domestic Abuse and Violence: Where Do Indian Women Stand? Available at: https://www.epw.in/engage/article/covid-19-domestic-abuse-and-violence-where-do. Accessed February 13.

38) Hossain MA, Sumi NS, Haque ME, Bari W. Consequences of intimate partner violence against women on under-five child mortality in Bangladesh Journal of interpersonal violence. 2014;29:1402-1417.

39) Drieskens S, Braekman E, Ridder KD, Gisle L, Charafeddine R, Hermans L, et al. Domestic violence during the COVID-19 confinement: do victims feel more socially isolated? Archives of Public Health. 2022 2022/01/25;80:39.

40) Sharma I, Pathak A. Women mental health in India Indian journal of psychiatry. 2015;57:S201-S204.

41) Tsai HM CYaWH. Health Issues in Taiwanese Women Austin J Womens Health. 2015;2(1)

42) Anjara SG, Brayne C, Van Bortel T. Perceived causes of mental illness and views on appropriate care pathways among Indonesians International journal of mental health systems. 2021;15:74.

43) Mumang AA. Gender Differences in Depression in the General Population of Indonesia: Confounding Effects. Depression research and treatment. 2021;2021.

44) Kieling $\mathrm{C}$, Baker-Henningham $\mathrm{H}$, Belfer $\mathrm{M}$, Conti $\mathrm{G}$, Ertem I, Omigbodun $\mathrm{O}$, et al. Child and adolescent mental health worldwide: evidence for action Lancet. 2011 Oct 22;378:1515-1525.

45) Fegert JM, Vitiello B, Plener PL, Clemens V. Challenges and burden of the Coronavirus 2019 (COVID-19) pandemic for child and adolescent mental health: a narrative review to highlight clinical and research needs in the acute phase and the long return to normality Child and Adolescent Psychiatry and Mental Health. 2020 2020/05/12;14:20.

46) Loades ME, Chatburn E, Higson-Sweeney N, Reynolds S, Shafran R, Brigden A, et al. Rapid Systematic Review: The Impact of Social Isolation and Loneliness on the Mental Health of Children and Adolescents in the Context of COVID-19 Journal of the American Academy of Child and Adolescent Psychiatry. 2020;59:1218-1239.e1213.

47) Duncan GJ, Yeung WJ, Brooks-Gunn J, Smith JR. How Much Does Childhood Poverty Affect the Life Chances of Children? American Sociological Review. 1998;63:406-423.

48) Zimmerman A, Lund C, Araya R, Hessel P, Sanchez J, Garman E, et al. The relationship between multidimensional poverty, income poverty and youth depressive symptoms: cross-sectional evidence from Mexico, South Africa and Colombia BMJ Glob Health. 2022 Jan;7.

49) Lund C, Cois A. Simultaneous social causation and social drift: Longitudinal analysis of depression and poverty in South Africa Journal of Affective Disorders. 2018 2018/03/15/;229:396-402.

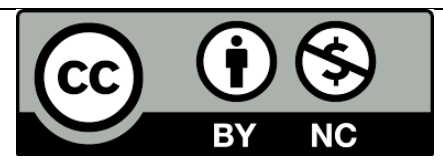

There is an Open Access article, distributed under the term of the Creative Commons Attribution - Non Commercial 4.0 International (CC BY-NC 4.0)

(https://creativecommons.org/licenses/by-nc/4.0/), which permits remixing, adapting and building upon the work for non-commercial use, provided the original work is properly cited. 